\title{
Síndrome metabólico y sus componentes en pacientes con síndrome de apnea-hipoapnea del sueño
}

\author{
Metabolic syndrome and its components in patients \\ with sleep apnea syndrome
}

\author{
M. Tumbeiro' ${ }^{1}$ A. Acuña ${ }^{1}$, M. T. Alves ${ }^{2}$, R. Dacal ${ }^{1}$, P. Marcos $^{1}$
}

\section{RESUMEN}

Fundamento. El síndrome de apnea-hipoapnea del sueño (SAHS) es un factor de riesgo reconocido de patología cardiovascular y la evidencia reciente sugiere que está relacionado con el síndrome metabólico (SM). El objetivo del estudio fue conocer la prevalencia de los componentes individuales del SM y del SM como entidad, en pacientes con sospecha clínica de SAHS, y ver si ambas entidades están en relación con la obesidad central.

Material y métodos. Se estudiaron prospectivamente a 486 pacientes consecutivos, 359 hombres (73,9\%), con una edad media de 57,3 $\pm 13,5$ años y un índice de masa corporal (IMC) de $32,1 \pm 6,5 \mathrm{~kg} / \mathrm{m} 2$ con sospecha clínica de SAHS. El SAHS se diagnosticó por registro nocturno. Un Índice de Apnea-Hipoapnea (IAH) > 5 era considerado diagnóstico de SAHS. El SM era diagnosticado siguiendo los criterios de la International Diabetes Federation.

Resultados. De los pacientes estudiados 325 (66,9\%) eran SAHS de grado moderado-grave. El IAH medio fue de $30,2 \pm 23,8$. De 456 pacientes $(93,8 \%)$ teníamos datos para el diagnóstico de SM y su prevalencia fue del $64,7 \%$ (295 pacientes). En el análisis multivariante, tanto la edad como el perímetro abdominal se comportaron como predictores de SAHS y de SM $(\mathrm{p}<0,05)$.

Conclusiones. El SM es más frecuente en pacientes con SAHS, y su prevalencia aumenta con su gravedad. La obesidad central, medida por el perímetro abdominal, resultó ser un factor predictor tanto de SAHS como de SM.

Palabras clave. Apnea del sueño. Síndrome metabólico. Obesidad abdominal.

\begin{abstract}
Background. Sleep Apnea Syndrome (SAHS) represents a significant risk factor for the development of cardiovascular disease and evidence suggests a relation with Metabolic Syndrome (MS). The aim of this study was to determine the prevalence of individual components of MS and of MS as an entity, in patients with clinical suspicion of SAHS, and their relation to central obesity.
\end{abstract}

Methods. We examined the records of 486 consecutive patients, 359 (73.9\%) men, with a mean age of $57.3 \pm 13.5$ years and a mean body mass index (BMI) of $32.1 \pm 6.5$ $\mathrm{kg} / \mathrm{m} 2$ ), with clinical suspicion of SAHS. SAHS was diagnosed from the nocturnal register. An Apnea-Hipopnea Index $(\mathrm{AHI})>5$ was considered SAHS. MS was evaluated according to the diagnostic criteria of the International Diabetes Federation.

Results. Three hundred and twenty-five $(66.9 \%)$ of the entire group were moderate-severe SAHS. The mean of AHI was $30.2 \pm 23.8$. We had sufficient data available on 456 patients (93.8\%) for MS diagnosis and its prevalence was $64.7 \%$ (295 patients). Multivariate analysis showed that age and abdominal perimeter were predictors of SAHS and MS ( $\mathrm{p}<0.05)$.

Conclusions. Patients with SAHS have a high prevalence of MS and their prevalence is greater in patients with major levels of SAHS. The rate of central obesity, measured by abdominal perimeter, predicts SAHS and MS.

Keywords. Sleep apnea. Metabolic syndrome. Abdominal obesity.
1. Complejo Hospitalario de Ourense. Servicio de Neumología.

2. Complejo Hospitalario de Ourense. Unidad de Apoyo a la Investigación.

Recepción: 17 de marzo de 2011

Aceptación provisional: 15 de abril de 2011

Aceptación definitiva: 22 de junio de 2011

\section{Correspondencia:}

Manuel Tumbeiro Novoa

Complejo Hospitalario de Orense

C/ Ramón Puga 52-54

32005 Ourense

E-mail: manuel.tumbeiro.novoa@sergas.es 


\section{INTRODUCCIÓN}

El síndrome de apnea-hipoapnea del sueño (SAHS) se caracteriza por episodios repetidos de obstrucción de la vía aérea superior, que se acompaña de desaturación nocturna, fragmentación del sueño y somnolencia diurna excesiva, objetivándose en estudios epidemiológicos una prevalencia en la población adulta del 2 al $4 \%^{1,2}$. Está asociado a un incremento de la morbi-mortalidad cardiovascular y cerebrovascular ${ }^{3,4}$. Sin embargo, el mecanismo exacto que relaciona la apnea del sueño y la morbilidad cardiovascular no está claro.

Según la Federación Internacional de Diabetes (IDF) se denomina síndrome metabólico (SM) o síndrome de resistencia a la insulina al conjunto de alteraciones metabólicas constituido por obesidad abdominal, disminución de la concentración de colesterol unido a las lipoproteínas de alta densidad (cHDL), elevación de la concentración de triglicéridos, aumento de la tensión arterial e hiperglucemia en ayunas ${ }^{5}$. La presencia de estas alteraciones metabólicas predicen un riesgo incrementado de enfermedad cardiovascular aterosclerótica y diabetes mellitus tipo 2 .

La asociación de SAHS y SM se conoce como síndrome $\mathrm{Z}$ desde la década de los años $90^{6}$, y hay evidencia clínica y experimental de que el SAHS puede iniciar el desarrollo o agravar la severidad de las alteraciones metabólicas individuales que forman la constelación del $\mathrm{SM}^{7}$; siendo la prevalencia del SM hasta un 40\% mayor en los pacientes con $\mathrm{SAHS}^{8}$. Por otro lado, el SM y sus componentes, en particular la obesidad central y la resistencia a la insulina/diabetes mellitus, puede influir en el desarrollo de SAHS; por esta interrelación algunos investigadores han propuesto que el SAHS puede ser un trastorno metabólico y un componente del $\mathrm{SM}^{9}$.

La presencia de SM en pacientes con SAHS es alta, si se compara con la población general ${ }^{10,11}$, y ello parece estar en relación con la obesidad, fundamentalmente la obesidad central, su frecuente asociación potencia la morbi-mortalidad cardiovascular que de forma individual tiene cada uno de ellos y se considera que se están convirtiendo en uno de los principales problemas de salud pública del siglo XXI a nivel mundial $^{5,12}$

El objetivo de este estudio fue evaluar la prevalencia del SM y sus componentes individuales en pacientes con sospecha clínica de SAHS remitidos a nuestro laboratorio de sueño, y ver si ambas entidades están en relación con la obesidad central.

\section{MATERIAL Y MÉTODOS}

Grupo de estudio. Se analizaron prospectivamente los pacientes remitidos a la consulta de la Unidad de Sueño con la sospecha clínica de SAHS en el periodo comprendido entre enero y diciembre del 2009. El 58\% de los pacientes eran remitidos desde atención primaria y el resto de atención especializada. Nuestra Unidad es referencia para los 335.642 habitantes adultos de la provincia de Ourense; dispone de 2 camas para la realización de estudios de polisomnografía convencional (PSG) con vigilancia nocturna (Somnostar alpha: Sensormedics, USA), y 4 polígrafos cardiorrespiratorios para estudios domiciliarios (PR) (dos Apnoscreen Pro, Erich Jaeger Gmbh \& Co. KG, Wurzburg, Germany y dos Embletta X100 - Home Sleep Study).

A todos los pacientes se les recogía en la historia clínica: datos epidemiológicos, antropométricos y clínicos; eran tallados y medidos, y se les calculaba su IMC. El perímetro del cuello era medido en centímetros (cm) a nivel de la membrana cricotiroidea y el perímetro de abdomen era medido en centímetros en el punto medio entre la última costilla y la cresta ilíaca y el ombligo.

Componentes del síndrome metabólico. La tensión arterial (TA) era medida el día de la prueba entre las 22 y 23 horas, tras 5 minutos en reposo y con el paciente en posición de sentado; se recogía la media de tres determinaciones separadas por 1-2 minutos usando un esfigmomanómetro digital (Omron M6 Confort).

Los datos analíticos se obtuvieron de la historia clínica informatizada del paciente, salvo los de aquellos que carecían de una 
analítica reciente (últimas 4 semanas). En este caso se realizaba la analítica a la mañana siguiente al registro en los pacientes estudiados con PSG o bien en Atención Primaria en los días posteriores al estudio (al grupo de registro poligráfico ambulatorio). Se estudiaban entre otras variables analíticas las necesarias para el diagnóstico de SM.

Diagnóstico de síndrome metabólico. Para el diagnóstico del SM seguimos los criterios de Clasificación de la International Diabetes Federation (IDF) ${ }^{13}$, que incluye: obesidad central (perímetro de cintura para europeos: varones, $>94 \mathrm{~cm}$, mujeres, $>80 \mathrm{~cm}$ ); y dos o más de los siguientes criterios: Triglicéridos $>150 \mathrm{mg} / \mathrm{dl}$ o tratamiento específico; Colesterol HDL $<40 \mathrm{mg} /$ $\mathrm{dl}$ (varones) o $<50 \mathrm{mg} / \mathrm{dl}$ (mujeres) o tratamiento específico; Presión Arterial > 130/85 mmHg o tratamiento específico; Glucemia basal > $100 \mathrm{mg} / \mathrm{dl}$ o diagnóstico previo de diabetes.

Diagnóstico de síndrome de apneahipoapnea del sueño. A todos los pacientes remitidos con sospecha clínica de SAHS se les realizó un registro nocturno: a 290 $(59,7 \%)$ una PR y a 196 (40,3\%) una PSG. Se repitieron estudios a 40 pacientes $(8,2 \%)$. Las definiciones de los eventos respiratorios y la evaluación de los registros nocturnos del sueño son los aconsejados por el Documento de Consenso de la SEPAR ${ }^{14}$. Apnea era definida como la ausencia o reducción mayor del $90 \%$ de la señal respiratoria durante más de 10 segundos. Hipoapnea era definida como una reducción discernible (mayor del 30\% y menor del 90\%) de la amplitud de la señal respiratoria de más de 10 segundos, acompañada de una caída de la saturación de la oxihemoglobina mayor del 3\% y/o un arousal electroencefalógrafico. Los datos eran expresados como índice de apnea-hipoapnea (IAH), basado en la suma del número de apneas e hipoapneas dividido por horas de sueño en la PSG, o por las horas de registro en la PR.

Todos los pacientes diagnosticados de SAHS tenían clínica compatible con dicha entidad (fundamentalmente ronquido noc- turno con pausas y/o hipersomnia diurna excesiva, pero a veces referían otra sintomatología como nicturia, ahogos nocturnos, despertares con sobresaltos o incluso insomnio) y un IAH $>5$. Para el propósito de este estudio, los pacientes eran divididos en tres subgrupos de severidad del SAHS, según el IAH, como sigue: SAHS de grado leve: IAH de 6 a 15; SAHS de grado moderado: IAH de 16 a 30 y SAHS grave si: IAH $>30$. Los pacientes con un $\mathrm{IAH} \leq 5$ eran considerados roncadores primarios.

Análisis estadístico. Inicialmente se realizó un análisis descriptivo de los datos; mediante las pruebas KolmogorovSmirnov y Shapiro-Wilk se comprobó la normalidad de las variables continuas. Estas variables fueron expresadas como media $\pm \mathrm{DE}$ (desviación estándar) y las variables categóricas como frecuencias y porcentajes. Para determinar las diferencias y comparar los distintos grupos de SAHS se utilizó el análisis de la varianza (ANOVA) para las variables continuas y el test Chi-cuadrado para las categóricas. Posteriormente se realizó un análisis de regresión univariante y multivariante para determinar aquellas variables predictoras de SM (regresión logística) y SAHS (regresión lineal). Para el análisis multivariante se tuvieron en cuenta las variables que resultaron significativas en el análisis univariante y aquellas clínicamente relevantes. Se consideraron significativos aquellos valores de $\mathrm{p}<0,05$. Para dicho análisis estadístico se utilizaron los programas SPSS 15.0 para Windows y el software libre R-2.10.1 -library mgcv.

\section{RESULTADOS}

Demográficos. Entre enero y diciembre de 2009, se estudiaron 486 pacientes de la población adscrita a la Unidad de Sueño del Complexo Hospitalario de Ourense, de los cuales 359 (73,9\%) eran hombres y 127 $(26,1 \%)$ mujeres. La edad media de los participantes fue de $57,3 \pm 13,5$ años (rango: 20-86); la media del IMC fue de $32,1 \pm 6,5$ $\mathrm{kg} / \mathrm{m}^{2}$, siendo obesos (IMC $\geq 30 \mathrm{~kg} / \mathrm{m}^{2}$ ) 295 pacientes $(60,7 \%)$, la media del perímetro 
de cuello fue de $42,6 \pm 4,6 \mathrm{~cm}$ y la media del perímetro de abdomen fue de 110,4 \pm $14,9 \mathrm{~cm}$. De 456 pacientes $(93,8 \%)$ se disponía de datos para el diagnóstico de SM. Las características clínicas y de laboratorio de nuestros pacientes se recogen en la tabla 1. La edad, el IMC, el perímetro del cuello y el perímetro abdominal aumentaban de forma significativa $(\mathrm{p}<0,001)$ con la gravedad del SAHS (Tabla 2).

Tabla 1. Características clínicas y de laboratorio de los pacientes de la serie

\begin{tabular}{lc}
\hline & $\mathrm{N}=486$ \\
Sexo masculino + & $359(73,9 \%)$ \\
Edad (años) * & $57,3 \pm 13,5$ \\
IMC (kg/m2) * & $32,1+6,5$ \\
Obesidad (IMC > 30) + & $295(60,7 \%)$ \\
P Cuello (cm) * & $42,6+4,6$ \\
P Abdomen (cm) * & $110,4+14,9$ \\
Tabaquismo actual o pasado + & $241(51 \%)$ \\
Alcohol + & $156(33,7 \%)$ \\
$\mathrm{H}^{\mathrm{a}}$ HTA + & $240(50,7 \%)$ \\
\hline Estudio del sueño & \\
PSG + & $196(40,3 \%)$ \\
PR + & $290(59,7 \%)$ \\
IAH * & $30,2 \pm 23,8$ \\
Sat OxiHB\% * & $92,4 \pm 3,5$ \\
CT90\% * & $20,9 \pm 3,6$ \\
\hline Laboratorio & \\
Glucemia (mg/dl) * & $109,8 \pm 28,2$ \\
Colesterol total (mg/dl) * & $195,9 \pm 40,0$ \\
cHDL (mg/dl) * & $47,8 \pm 14,2$ \\
Triglicéridos (mg/dl) * & $132,8 \pm 92,0$ \\
Apo A * & $145,7 \pm 26,8$ \\
Apo B * & $113,8 \pm 28,8$ \\
Microalbumina * & $30,5 \pm 72,4$ \\
Ac úrico (mg/dl) * & $5,9 \pm 1,5$ \\
Fibrinógeno (mg/dl) * & $396,8 \pm 94,2$ \\
\hline SM y sus componentes & $\mathrm{N}=456$ \\
SM (sí) & $64,7 \%$ \\
Diabetes (sî) & $60,0 \%$ \\
HTA (sí) & $59,0 \%$ \\
cHDL (sí) & $56,0 \%$ \\
Triglicéridos (sí) & $30,0 \%$ \\
\hline + No pacientes (porcentaje sobre el total); * media $\pm \mathrm{DE}$ \\
\end{tabular}


Tabla 2. Características clínicas y de laboratorio de los pacientes según la gravedad del SAHS y de los controles.

\begin{tabular}{|c|c|c|c|c|c|}
\hline & $\begin{array}{c}\text { Roncadores } \\
\text { primarios }\end{array}$ & Leve & Moderado & Grave & \\
\hline$N=486$ & $56(11,5 \%)$ & $105(21,6 \%)$ & $131(26,9 \%)$ & $194(39,9 \%)$ & p Valor \\
\hline Edad (años) & $49,5 \pm 15,5$ & $53,5 \pm 14,8$ & $59,1 \pm 12,1$ & $60,4 \pm 11,8$ & $<0,001$ \\
\hline $\operatorname{IMC}\left(\mathrm{kg} / \mathrm{m}^{2}\right)$ & $28,6 \pm 5,2$ & $30,2 \pm 6,2$ & $30,8 \pm 4,4$ & $34,9 \pm 7,0$ & $<0,001$ \\
\hline IAH & $3,9 \pm 3,2$ & $10,3 \pm 3,3$ & $22,6 \pm 4,4$ & $53,6 \pm 19,8$ & $<0,001$ \\
\hline Perímetro cuello (cm) & $38,5 \pm 4,4$ & $41,1 \pm 3,8$ & $42,4 \pm 4,2$ & $44,7 \pm 4,1$ & $<0,001$ \\
\hline Perímetro abdomen (cm) & $100,2 \pm 14,4$ & $104,5 \pm 14,4$ & $108,5 \pm 11,1$ & $117,7 \pm 14,3$ & $<0,001$ \\
\hline Sat OxiHb \% & $95,3 \pm 2,3$ & $93,0 \pm 26$ & $93,0 \pm 2,6$ & $90,6 \pm 3,9$ & $<0,001$ \\
\hline СТ $90 \%$ & $3,6 \pm 11,8$ & $11,1 \pm 23,0$ & $13,0 \pm 23,0$ & $34,9 \pm 32,1$ & $<0,001$ \\
\hline \multicolumn{6}{|l|}{ Valores de laboratorio } \\
\hline Glucemia (mg/dl) & $99,9 \pm 21,2$ & $105,4 \pm 26,2$ & $110,3 \pm 25,9$ & $113,8 \pm 30,6$ & 0,005 \\
\hline Colesterol (mg/dl) & $193,4 \pm 36,9$ & $191,0 \pm 36,5$ & $199,3 \pm 39,5$ & $196,0 \pm 43,2$ & 0,498 \\
\hline c HDL (mg/dl) & $51,8 \pm 14,3$ & $47,1 \pm 11,4$ & $47,5 \pm 14,3$ & $47,1 \pm 15,3$ & 0,237 \\
\hline Triglicéridos (mg/dl) & $122,2 \pm 106,3$ & $112,7 \pm 69,8$ & $130,7 \pm 66,4$ & $145,9 \pm 107,5$ & 0,03 \\
\hline
\end{tabular}

IMC = índice de masa corporal; $\mathrm{IAH}=$ índice de apnea-hipoapnea; Sat OxiHb\%= saturación de la oxihemoglobina en \%; CT90\%= Tiempo del registro en que la saturación de la oxihemoglobina es menor del $90 \%$; cHDL= colesterol unido a las lipoproteínas de alta densidad

Estudio de sueño. A 290 (59,7\%) pacientes se les realizó una PR ambulatoria y a 196 (40,3\%) una PSG. No se encontraron diferencias significativas en las características clínicas ni en los datos de laboratorio entre aquellos pacientes a los que se les realizó una PSG respecto a los de PR; sin embargo, sí se detectaron diferencias en los eventos respiratorios (IAH) y en la saturación de la oxihemoglobina.

El IAH medio fue de $30,2 \pm 23,8$. Tenían un $\mathrm{IAH}>5,430$ pacientes $(88,5 \%)$, siendo SAHS leve 105 pacientes $(21,6 \%)$, SAHS moderado $131(26,9 \%)$ y SAHS grave 194 $(39,9 \%)$. Los otros 56 pacientes $(11,5 \%)$ tenían un IAH $\leq 5$ y eran considerados roncadores primarios.

Como podemos ver en la tabla 2 , la saturación media de la oxihemoglobina y el tiempo de registro con una saturación menor de $90 \%$ (CT90\%) empeoraron de forma significativa a medida que aumentaba la gravedad del SAHS $(\mathrm{p}<0,001)$.
Componentes del SM. Del total de los 486 pacientes, se obtuvieron datos para poder diagnosticar SM en 456 (93,8\%); de ellos 295 (64,7\%) cumplían criterios diagnósticos de SM. Como se ve en la tabla 2, el tanto por ciento de pacientes con obesidad central medida por el perímetro abdominal, aumentó de forma significativa al aumentar la gravedad del SAHS ( $\mathrm{p}<$ $0,001)$.

Los componentes individuales del SM así como sus niveles plasmáticos aumentaban con la gravedad del SAHS, aunque no en todos de forma significativa. La presencia de hiperglucemia y de HTA aumentaban de forma significativa con la severidad del SAHS ( $<$ < 0.001); en los otros dos componentes del SM, hipertrigliceridemia y disminución de los niveles colesterol HDL existía una tendencia positiva, pero sin llegar a la significación estadística ( $\mathrm{p}=0,236, \mathrm{p}=0,591$, respectivamente) (Tabla 3 ). 
Tabla 3. Resultado de los análisis de regresión multivariante

\begin{tabular}{c|cc|cc}
\hline & IAH (SAHS) & \multicolumn{2}{c}{ SM } \\
\hline Variables & $\beta$ (IC 95\%) & $\mathbf{p}$ & OR (IC 95\%) & p \\
\hline Sexo (hombre) & $5,78(3,03-11,03)$ & $<0,001$ & $0,79(0,45-1.38)$ & 0,352 \\
Edad (años) & $1,045(1,02-1,07)$ & 0,001 & $1,04(1,02-1,06)$ & $<0,001$ \\
Abdomen (cm) & $1,03(1,00-1,05)$ & 0,028 & $1,085(1,06-1,11)$ & - \\
SM & $0,76(0,36-1,61)$ & 0,474 & - & 0,001 \\
IAH & - & - & $0,841(0,40-1,76)$ & 0,645 \\
\hline
\end{tabular}

IAH= Índice apnea hipoapnea.Variable respuesta regresión lineal;SM= Síndrome metabólico. Variable respuesta regresión logística; $\beta=$ Beta- coeficiente de regresión; IC= Intervalo de confianza para el coeficiente de regresión al 95\% p= p-valor; R2 = r-cuadrado (Para la regresión logística hemos usado el R- cuadrado de Nagelkerke); OR= odds ratio.

El número de componentes del SM se incrementa de $1,6 \pm 1,3$ en el subgrupo de los roncadores primarios a $2,3 \pm 1,0$ en el subgrupo de SAHS grave $(\mathrm{p}<0,001)$. El SM como entidad se incrementa con la gravedad del SAHS de forma significativa pasando del 49,0 \% en los roncadores primarios al $75,8 \%$ en el grupo de SAHS grave $(\mathrm{p}<0,001)$. (Fig 1).

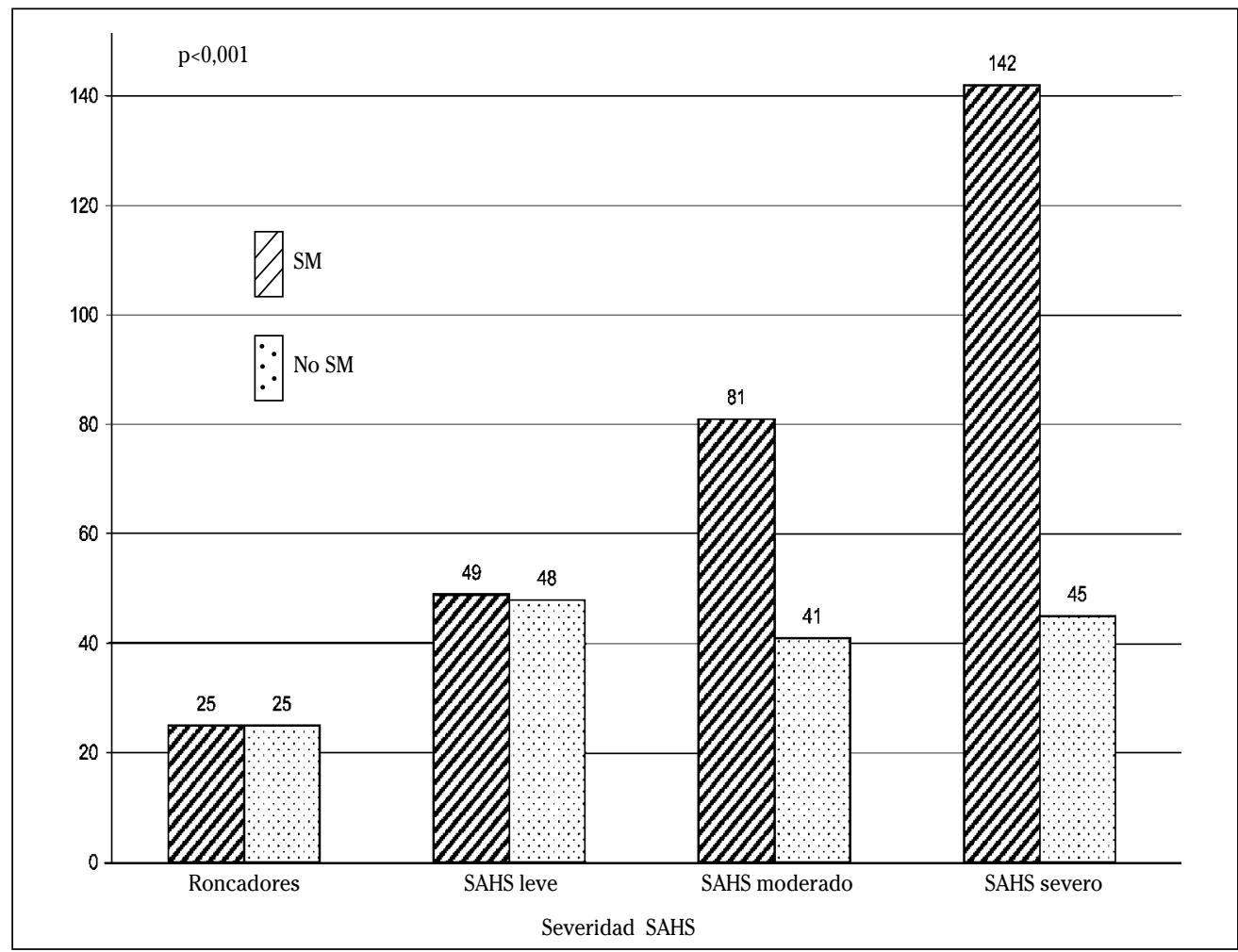

Figura 1. Incremento del SM en función del SAHS. 
Como se puede ver en la figura 2 , la mediana del perímetro abdominal aumenta progresivamente con la gravedad de SAHS y dichas medianas son mayores en el grupo de pacientes con SM que en los pacientes sin SM.

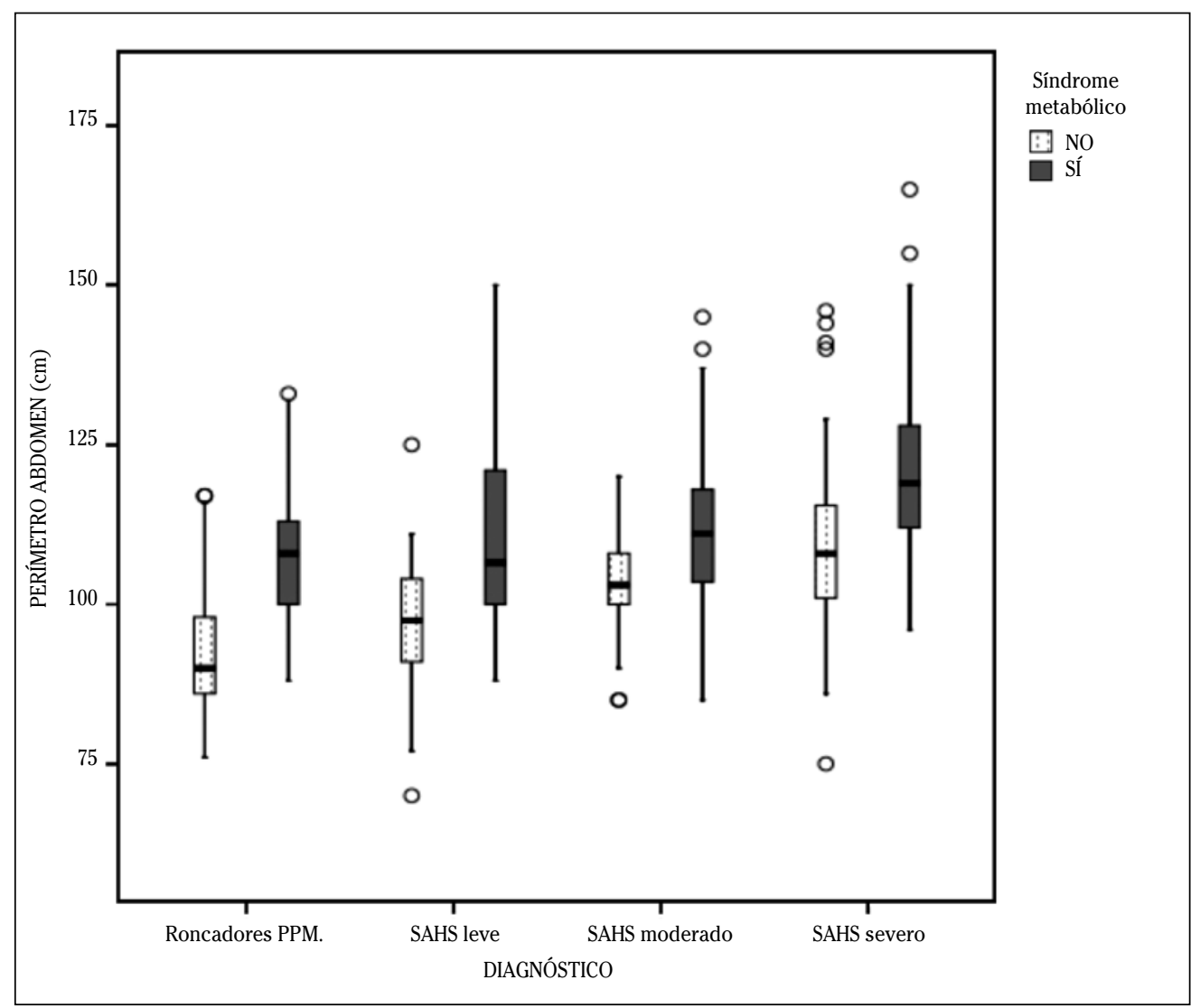

Figura 2. Relación entre perímetro abdominal, síndrome metabólico y severidad del SAHS.

En el análisis univariante, tanto la presencia de SAHS como de SM se relacionan de forma significativa entre otras variables con: la edad $(\mathrm{p}<0,001)$; perímetro de cuello $(\mathrm{p}<0,001)$; perímetro de abdomen $(\mathrm{p}<0,001)$; IMC $(\mathrm{p}<0,001)$; saturación media de la oxihemoglobina ( $\mathrm{p}<0,001)$; СТ90\% $(\mathrm{p}<0,001)$ y presencia de diabetes $(\mathrm{p}<0,008$; $\mathrm{p}<0,001)$ ).

En el análisis multivariante, resultaron factores predictores de SAHS: sexo varón $(\mathrm{p}<0,001)$, la edad $(\mathrm{p}<0,001)$ y el perímetro del abdomen $(\mathrm{p}=0,028)$; siendo factores predictores de SM sólo la edad $(\mathrm{p}<0,001)$ y el perímetro de abdomen $(\mathrm{p}<0,001)$ (Tabla 3).

\section{DISCUSIÓN}

Los principales hallazgos de nuestro estudio, realizado a pacientes con sospecha clínica de SAHS son: la presencia de una alta prevalencia del SM y sus componentes individuales en nuestros pacientes con SAHS; esta prevalencia del SM y de sus componentes aumenta con la severidad del SAHS determinado por el IAH. También se ha observado que los componentes del SM 
más prevalentes en nuestros pacientes con SAHS son la HTA y la hiperglucemia; y los análisis multivariantes, reflejan que el perímetro abdominal es un factor predictor tanto de un mayor IAH como de la presencia de SM.

Estudios previos demuestran que los pacientes con SAHS tienen de 3 a 9 veces más riesgo de tener SM; y por otro lado, la presencia de SM ocurre del 50 al 85\% de los pacientes con SAHS ${ }^{8,15,16}$. Nosotros, y considerando que los pacientes fueron diagnosticados de SAHS si el IAH era mayor a 5 , encontramos una alta prevalencia de SM en todo el grupo, que aumentaba hasta el $75,8 \%$ en los pacientes con SAHS grave. Llama la atención la elevada prevalencia del SM en los sujetos sin SAHS y en los SAHS de grado leve de nuestra muestra (49\%) en comparación con la de la población general que se cifra entre el 7 y el $11 \%^{10,11}$. Este hecho puede deberse a la media de edad alta de nuestros pacientes y a su obesidad, al ser una población muy seleccionada; cuando en la serie de Gutiérrez $\mathrm{J}$ y col ${ }^{10} \mathrm{se}$ analizaban los pacientes obesos encontraban una prevalencia de SM del 46,5\% de los sujetos.

Además de esta mayor prevalencia de SM en pacientes con SAHS grave, también se observó una mayor frecuencia en los componentes individuales igual que en estudios previos ${ }^{17,18}$, y el número de éstos aumentó con la severidad del SAHS.

La enfermedad cardiovascular, especialmente la HTA ha sido asociada con el SAHS. El Sleep Heart Study ${ }^{19}$ demuestra que el SAHS es un factor de riesgo independiente para la HTA y la enfermedad cardiovascular. En nuestros pacientes la HTA está presente en el 59\% de todo el grupo, llegando a una prevalencia del $67 \%$ en los diagnosticados de SAHS grave.

Por otro lado, hay cada vez más evidencia de que el SAHS se asocia con resistencia a la insulina y a la alteración del metabolismo de la glucosa, de forma independiente a la presencia de obesidad ${ }^{20,21}$. La hiperglucemia en ayunas es el componente del SM más prevalente en los pacientes de nuestra serie, llegando a un $71 \%$ en los pacientes con SAHS grave.
El SAHS puede conducir no sólo a cambios cuantitativos en el nivel de lípidos plasmáticos, sino también a cambios cualitativos. En el Sleep Heart Health Study ${ }^{22}$ los niveles de colesterol total no variaban con los cambios del IAH, pero hay un relación inversa entre los niveles de colesterol-HDL y el IAH, así como con la elevación de los triglicéridos plasmáticos sobre todo en adultos jóvenes. Nuestros pacientes presentaron un nivel de colesterol-HDL bajo en el $56 \%$ de los casos, y sólo un 30\% tenían hipertrigliceridemia, hallazgos similares a los encontrados por Peled y col ${ }^{17}$.

Como es bien sabido, la presencia de obesidad, fundamentalmente la obesidad abdominal o central, que en la práctica clínica tiene una buena correlación con la medida del perímetro abdominal ${ }^{23}$, es uno de los factores de riesgo más importante para la aparición del $\mathrm{SAHS}^{24}$, además de asociarse a un aumento de resistencia a la insulina, componente básico del $\mathrm{SM}^{25}$. En el análisis multivariante de nuestra serie de pacientes, junto con la edad, la única variable que tiene una relación estadísticamente significativa con el síndrome de apnea-hipoapnea del sueño y el síndrome metabólico, es el perímetro abdominal como reflejo de la obesidad abdominal. La relación de la obesidad abdominal con el SM, al ser un criterio necesario para su diagnóstico si seguimos la definición de la IDF, está clara; si bien, existe controversia en la literatura de si es el IMC o el perímetro abdominal el mejor factor predictor de la aparición del SAHS. Nuestros datos sugieren que el acúmulo de grasa visceral es un factor de riesgo importante para la presencia de SAHS; hallazgos similares al nuestro aparecen en otras series ${ }^{21,25,26}$, mientras que en otros estudios es el IMC el factor relacionado con la presencia de $\mathrm{SAHS}^{22}$.

Este estudio puede tener importantes aplicaciones prácticas. En los pacientes con SAHS que vemos en nuestras unidades de sueño, sobre todo si presentan un SAHS de grado moderado y grave, debemos de excluir de manera rutinaria la presencia de los diferentes componentes y del síndrome metabólico como entidad; y como estos pacientes suelen tener sobrepeso evidente o 
incluso obesidad, debemos de lograr que reduzcan su peso, pues incluso una reducción de peso modesta (del cinco al diez por ciento del peso total) puede ser beneficiosa, siendo esto un pilar fundamental como ya han demostrado diversos trabajos para el manejo del SAHS independiente de su grado de severidad ${ }^{27,28}$, evidentemente, además de comenzar en los casos indicados tratamiento con presión positiva continua de la vía aérea (CPAP). Modificaciones saludables en el estilo de vida, en especial la reducción del peso y el aumento de la actividad física, son también la estrategia central del tratamiento del $\mathrm{SM}^{29,30}$.

En nuestro estudio encontramos que los pacientes a los que se les realizó una PR presentaban un IAH significativamente más alto y una menor saturación de la oxihemoglobina que a los que se les realizó una PSG. Una explicación a esto podría ser el hecho de que a los pacientes con alta sospecha clínica de SAHS y con importante afectación clínica se les realizaba una poligrafía respiratoria, debido a la mayor disponibilidad de polígrafos respiratorios. Esto podría haber originado que se haya realizado sistemáticamente una $\mathrm{PR}$ a los pacientes más graves.

En conclusión, en los pacientes con SAHS es frecuente encontrar los componentes del SM presentes y la entidad propiamente dicha. A mayor obesidad central, medida por el perímetro abdominal más frecuente es la presencia del SAHS y del $\mathrm{SM}$, por lo que en estos pacientes es básico conseguir la corrección de su exceso de peso.

\section{Agradecimientos}

A Laura González Dacal, Generosa Rodríguez Fernández y José Luis Barge Bello (técnicos de la Unidad del Sueño) por su ayuda en la recogida de datos.

\section{BIBLIOGRAFÍA}

1. Durán J, Esnaola S, Rubio R, Iztueta A. Obstructive sleep apnea-hypoapnea and related clinical features in a population-based sample of subjects aged 30 to 70 years. Am J Respir Crit Care Med 2001; 163:685-689.

2. Young T, Palta M, Dempsey J, Skatrud J, Weber S, BADR S. The occurrence of sleep disordered breathing among middle-aged adults. N Engl J Med 1993; 328:1230-1235.

3. Marín JM, Carrizo SJ, Vicente E, Agustí AGN. Long-term cardiovascular outcomes in men with obstructive sleep apnoea-hypopnoea with or without treatment with continuous positive airway pressure: an observational study. Lancet 2005; 365:1046-1053.

4. LAVIE P. Mortality in sleep apnoea sydrome: a review of the evidence. Eur Respir Rev 2007; 16: 203-210.

5. Eckel RH, Grundy SM, Zimmet PZ. The metabolic syndrome. Lancet 2005; 365: 1415-1428.

6. Wilcox I, McNamara SG, Coluins FL, Grunstein RR, Sullivan CE. Syndrome Z: the interaction of sleep apnoea, vascular risk factors and heart disease. Thorax 1998; 53: S25-S28.

7. TASALI E, IP MSM. Obstructive sleep apnea and Metabolic Syndrome: Alterations in glucose metabolism and inflammation. Proc Am Thorac Soc 2008; 5: 207-217.

8. Coughlin S, Mawdsley L, Mugarza J, Calverley P, WILDING J. Obstructive sleep apnoea is independently associated with an increased prevalence of metabolic syndrome. Eur Heart J 2004; 25: 735-741.

9. Vgontzas AN, Bixler EO, Chrousos GP. Sleep apnea is a manifestation of the metabolic syndrome. Sleep Med Rev 2005; 9: 211-224.

10. Gutiérrez J, López JJ, RodríGuez J, Garcés C, LloRENS MT. Prevalencia del síndrome metabólico en la población laboral. El Corazón de Asepeyo. An Med Interna (Madrid) 2008; 25: 325-330.

11. Alegría E, Cordero A, Laclaustra M, Grima A, León M, CASASnovas JA, et al. Prevalencia del síndrome metabólico en la población laboral española: registro MESYAS. Rev Esp Cardiol 2005; 58: 797-806.

12. BRAY GA. Medical consequences of obesity. J Clin Endocrinol Metab 2004; 89: 2583-2589.

13. Alberti KG, Zimmet P, Shaw J. IDF Epidemiology Task Force Consensus Group. The metabolic syndrome: a new worldwide definition. Lancet 2005; 366: 1059-1062.

14. Consenso Nacional sobre Síndrome de Apneas-Hipoapneas del Sueño. Definición y concepto, fisiopatología, clínica y exploración del SAHS. Arch Bronconeumol 2005; 41 Supl 4: 12-29. 
15. Kono M, Tatsumi K, Saibara T, Nakamura A, TANABE N, TAKIGUCHI $Y$ et al. Obstructive sleep apnea syndrome is associated with some components of metabolic syndrome. Chest 2007; 131: 1387-1392.

16. Gruber A, Horwood F, Sithole J, Ali NJ, Idris I. Obstructive sleep apnoea is independently associated with the metabolic syndrome but not insulin resistance state. Cardiovasc Diabetol 2006; 5: 22.

17. Peled N, Kassirer M, Shitrit D, Kogan Y, Shlomi D, BERLINER AS et al. The association of OSA with insulin resistance, inflammation and metabolic syndrome. Respir Med 2007; 101: 1696-1701.

18. LAM JCM, LAM B, LAM C-L, Fong D, WANG JKL, TsE H-F et al. Obstructive sleep apnea and the metabolic syndrome in communitybased Chinese adults in Hong Kong. Respir Med 2006; 100: 980-987.

19. Shahar E, Whitney CW, Redline S, Lee ET, Newman $\mathrm{AB}$, NiETo J et al. Sleep-disordered breathing and cardiovascular disease. Cross-sectional results of the Sleep Heart Health Study. Am J Respir Crit Care Med 2001; 163: 19-25.

20. IP MSM, LAM B, NG MMT, Lam WK, Tsang KWT, LAM KSL. Obstructive sleep apnea is independently associated with insulin resistance. Am J Respir Crit Care Med. 2002; 165: 670676.

21. Vgontzas AN, Bixler ED, Chousos GP. Metabolic disturbances in obesity versus sleep apnea: the importance of visceral obesity and insulin resistance. J Internn Med 2003; 254: 32-44.

22. Newman AB, Nieto FJ, Guidry U, Lind BK, Redline $\mathrm{S}$, SHAHAR E et al. Relation of sleep-disordered breathing to cardiovascular disease risk factors: The Sleep Heart Health Study. Am J Epidemiol 2001; 154: 50-59.
23. Foster GD. Principles and practices in the management of obesity. Am J Respir Crit Care Med 2003; 168: 274-280

24. Strohl EP, Redline S. Recognition of obstructive sleep apnea. Am J Respir Crit Care Med 1996; 154: 279-289

25. Shinohara E, Kihara S, Yamashita S, Yamane M, NishidA M, ARAI T et al. Visceral fat accumulation as an important risk factor for obstructive sleep apnoea syndrome in obese subjects. J Intern Med 1997; 241: 11-18.

26. Martínez-Rivera C, Abad J, Fiz JA, Rios J, MoreRA J. Usefulness of truncal obesity indices as predictive factors for obstructive sleep apnea síndrome. Obesity 2008; 16: 113-118

27. Tuomilehto HPI, Seppä JM, Partinen MM, Petonen M, Gylling H, TuOmilehto JOI, et al. Lifestyle intervention with weight reduction: Firs-line treatment in mild obstructive sleep apnea. Am J Respir Crit Care Med 2009; 179: 320-327.

28. Johansson K, Neovius M, Lagerros YT, Harlid R, RÖSSNER S, GRANAT F et al. Effect of a very low energy diet on moderate and severe obstructive sleep apnoea in obese men: a randomised controlled trial. Brit Med J 2009; 339: b4609.

29. Orchard TJ, Temprosa M, Goldberg R, Haffner S, Ratner R, Marcovina $S$ et al. Diabetes Prevention Program Research Group. The effect of metformin and intensive lifestyle intervention on the metabolic syndrome: the Diabetes Prevention Program randomized trial. Ann Intern Med 2005; 142: 611-619.

30. Lindström J, Louheranta A, Mannelin M, Rastas M, SAlminen V, ERIKSSON J et al. The Finnish Diabetes Prevention Study (DPS): Lifestyle intervention and 3-year results on diet and physical activity. Diabetes Care 2003; 26: 3230-3236. 